\title{
ON BRESSAN'S CONJECTURE ON MIXING PROPERTIES OF VECTOR FIELDS
}

\author{
STEFANO BIANCHINI \\ SISSA-ISAS \\ via Beirut 2-4, I-34014, Trieste, Italy \\ E-mail: bianchin@sissa.it
}

\begin{abstract}
In [9], the author considers a sequence of invertible maps $\mathbf{T}_{i}: S^{1} \rightarrow S^{1}$ which exchange the positions of adjacent intervals on the unit circle, and defines as $A_{n}$ the image of the set $\{0 \leq x \leq 1 / 2\}$ under the action of $\mathbf{T}_{n} \circ \ldots \circ \mathbf{T}_{1}$,

$$
A_{n}=\left(\mathbf{T}_{n} \circ \ldots \circ \mathbf{T}_{1}\right)\left\{x_{1} \leq 1 / 2\right\} .
$$
\end{abstract}

Then, if $A_{n}$ is mixed up to scale $h$, it is proved that

$$
\sum_{i=1}^{n}\left(\operatorname{Tot} . \operatorname{Var} .\left(\mathbf{T}_{i}-\mathbf{I}\right)+\operatorname{Tot} . \operatorname{Var} .\left(\mathbf{T}_{i}^{-1}-\mathbf{I}\right)\right) \geq C \log \frac{1}{h} .
$$

We prove that (2) holds for general quasi incompressible invertible BV maps on $\mathbb{R}$, and that this estimate implies that the map $\mathbf{T}_{n} \circ \ldots \circ \mathbf{T}_{1}$ belongs to the Besov space $B^{0,1,1}$, and its norm is bounded by the sum of the total variation of $\mathbf{T}-\mathbf{I}$ and $\mathbf{T}^{-1}-\mathbf{I}$, as in (2).

1. Introduction. The existence of solutions for the transport equation

$$
u_{t}+a(t, x) \cdot \nabla u=0, \quad(t, x) \in \mathbb{R}^{+} \times \mathbb{R},
$$

is an important topic of research. In the paper of DiPerna-Lions [13], the notion of renormalized solution is introduced, for measurable vector fields $a(t, x)$ with bounded divergence. It is then proved that the solution to (3) are renormalized if the vector field $a(t, x)$ belongs to the Banach space $L^{1}\left((0, T) ; W^{1, p}\right)$ (with some bound on the exponential growth of the trajectories). As a consequence there is a unique solution to (3) with bounded initial data, which depends continuously in $L^{1}$, and it is possible to define a flow $X: \mathbb{R} \times \mathbb{R}^{n} \rightarrow \mathbb{R}^{n}$ for the discontinuous ODE

$$
\frac{d X}{d t}=a(t, X)
$$

2000 Mathematics Subject Classification: 35F25.

Key words and phrases: transport equation, Besov spaces, mixing properties.

The author would like to thank A. Bressan and K. Zumbrun for their suggestions.

The paper is in final form and no version of it will be published elsewhere. 
Recently, in [1], Ambrosio extended the result to vector fields a which are only BV. To have a panoramic view of the recent developments, see $[4,6,7,10,12,15]$.

A possible new direction of research of this space has been considered by Bressan $[8,9]$. In the first paper [8], he showed the ill posedness of the $n \times n$ system of conservation laws in 2 space dimensions

$$
u_{t}+\sum_{i=1}^{m} \partial_{x_{i}}\left(f_{i}(|u|) u\right)=0, \quad u \in \mathbb{R}^{n},
$$

if $|u| \in L^{\infty}$. If we set $\rho=|u|, \theta=u / \rho$, the system can be rewritten as

$$
\begin{gathered}
\rho_{t}+\sum_{i=1}^{m} \partial_{x_{i}}\left(f_{i}(\rho) \rho\right)=0, \\
\theta_{t}+\sum_{i=1}^{m} f_{i}(\rho) \partial_{x_{i}} \theta=\theta_{t}+a(t, x) \cdot \nabla \theta=0 .
\end{gathered}
$$

It is conjectured that if $|u|$ is in $\mathrm{BV}_{\text {loc }}$ and $1 / C<|u|<C$ (by [14] this space is invariant for the flow of (5)), then there exists a solution to the flow for the ODE

$$
\frac{d X}{d t}=a(t, X)
$$

associated to the transport equation (6). This problem has been solved in [2, 3], using the theory of renormalized solutions. In [11] it is shown that the flow generated by (6) is not in the space BV.

A different approach to construct such a flow is given in [9]. The basic idea is to consider on $S^{1}$ a set $A$ such that

$$
c \leq \frac{1}{2 h} \int_{x-h}^{x+h} \chi_{A}(y) d y \leq(1-c),
$$

where $c$ is a fixed constant in $(0,1)$. The sets satisfying the above condition are said to be mixed up to scale $h$, which means that in all segments of size $2 h$ there is at least a subset of $A$ of total length greater than $2 c h$ and lower than $2(1-c) h$. Together with these sets, the author consider the maps

$$
\mathbf{T}_{\bar{x}, a, b}=\mathbf{I}+ \begin{cases}a, & \bar{x}-b<x \leq \bar{x} \\ b, & \bar{x}<x \leq \bar{x}+a, \\ 0, & \text { otherwise }\end{cases}
$$

The total variation of $\mathbf{T}-\mathbf{I}$ is $2(a+b)$. Equivalently, in the language of [9], we can say that the cost of exchanging positions to two adjacent segments $(\bar{x}-b, \bar{x}),(\bar{x}, \bar{x}+a)$ is $2(a+b)$.

Bressan proves the following estimate: if after applying a finite number of maps $\mathbf{T}_{\bar{x}_{i}, a_{i}, b_{i}}$, we obtain that

$$
\left(\mathbf{T}_{\bar{x}_{n}, a_{n}, b_{n}} \circ \ldots \circ \mathbf{T}_{\bar{x}_{1}, a_{1}, b_{1}}\right) \chi_{A}= \begin{cases}1, & 0 \leq x \leq|A|, \\ 0, & \text { otherwise }\end{cases}
$$

then the total variation of the maps satisfies the lower bound

$$
\sum_{i=1}^{n} \operatorname{Tot.Var} .\left(\mathbf{T}_{\bar{x}_{i}, a_{i}, b_{i}}-\mathbf{I}\right)=\sum_{i=1}^{n} 2\left(a_{i}+b_{i}\right) \geq \mathcal{O}(1) \log \frac{1}{h} .
$$


The author considers a smooth vector field $v(t, x)$, and its corresponding flux $X(t, x)$,

$$
\frac{d X}{d t}=v(t, X), \quad X(0, x)=x
$$

satisfying the quasi incompressibility condition

$$
\frac{1}{C} \leq J(t, x)=\operatorname{det} \nabla_{x} X(t, x) \leq C
$$

for some fixed $C>0$. If one assumes that the flow mixes up to size $h$, i.e. the image of the unit ball is mapped to a set $A$ such that

$$
c \leq \frac{1}{|B(0, h)|} \int_{B(x, h)} \chi_{A}(y) d y \leq(1-c),
$$

where $|A|$ is the Lebesgue measure of the set $A$, then the conjecture is that the vector field $v$ satisfies

$$
\int_{0}^{T} \operatorname{Tot} . \operatorname{Var} .(v(t)) d t \geq \mathcal{O}(1) \log \frac{1}{h} .
$$

We can interpret the above conjecture as an estimate of $L^{1}$ compactness of characteristic functions under the action of the quasi incompressible vector fields. A different approach is to find a Banach space $\mathcal{B}$ such that we can rewrite the estimate (10) in the form

$$
\left\|\chi_{\mathbf{T} A}\right\|_{\mathcal{B}} \leq\left\|\chi_{A}\right\|_{\mathcal{B}}+\kappa\left(\operatorname{Tot} . \operatorname{Var} .(\mathbf{T}-\mathbf{I})+\operatorname{Tot} . \operatorname{Var} .\left(\mathbf{T}^{-1}-\mathbf{I}\right)\right),
$$

for all maps $\mathbf{T}: \mathbb{R}^{n} \rightarrow \mathbb{R}^{n}$ quasi incompressible and $\kappa>0$. Moreover, for sets mixed up to scale $h$, we have the estimate

$$
\left\|\chi_{B}\right\|_{\mathcal{B}} \geq \kappa^{\prime} \log \frac{1}{h}
$$

In this paper we show for the one-dimensional case a space with the above characteristics is the space $\mathcal{B}$ is the homogeneous Besov space $\dot{B}^{0,1,1}$, which is defined by the norm

$$
\|u\|_{\dot{B}^{0,1,1}}=\int_{0}^{1} \frac{1}{h} \sup _{|t| \leq h}\left\{\int_{\mathbb{R}^{n}}|u(x+t)-u(x)| d x\right\} d h .
$$

For an introduction to Besov spaces see [16]. Here we only observe that we can define the general Besov space $B^{s, p, q}$ as

$$
\|u\|_{B^{s, p, q}}=\left(\int_{0}^{1} \frac{1}{h^{s q}}\left(\sup _{|t| \leq h} \int_{\mathbb{R}^{n}}|u(x+t)-u(x)|^{p} d x\right)^{q / p} \frac{d h}{h}\right)^{1 / q},
$$

so that we see in the case under consideration that $s=0, p=q=1$.

As a consequence, Bressan's conjecture on mixing properties of vector fields can be stated as follows: if $X: \mathbb{R}^{+} \times \mathbb{R}^{n} \rightarrow \mathbb{R}^{n}$ is the flow of (11) satisfying (12), then

$$
\|X(t)-\mathbf{I}\|_{\dot{B}^{0,1,1}} \leq C \int_{0}^{t} \operatorname{Tot.Var} .(v(s)) d s .
$$

The paper is organized as follows. 
In Section 2, we introduce some notations and definitions on the quasi incompressible invertible BV maps. We then define a functional $\mathcal{P}(A)$ on measurable sets of the real line,

$$
\mathcal{P}(A)=\int_{0}^{1} \frac{1}{h} \int_{\mathbb{R}}\left|\chi_{A}(x)-\frac{1}{2 h} \int_{x-h}^{x+h} \chi_{A}(y) d y\right| d x d h,
$$

and show that, under the assumption of quasi incompressibility, there is an equivalent formulation where the maps are measure preserving.

In Section 3, we prove that $\mathcal{P}(A)$ satisfies (15), i.e.

$$
\mathcal{P}(\mathbf{T} A) \leq \mathcal{P}(A)+\kappa\left(\operatorname{Tot} . \operatorname{Var} .(\mathbf{T}-\mathbf{I})+\operatorname{Tot} . \operatorname{Var} .\left(\mathbf{T}^{-1}-\mathbf{I}\right)\right),
$$

and moreover for sets mixed up to scale $h$ the estimate (16) holds. To be more precise, the proof shows that in our one-dimensional setting,

$$
\mathcal{P}(\mathbf{T} A) \leq \mathcal{P}(A)+\mathcal{O}(1)\left(\text { jump part of }(\mathbf{T}-\mathbf{I}),\left(\mathbf{T}^{-1}-\mathbf{I}\right)\right) .
$$

Thus only the jumps of $\mathbf{T}, \mathbf{T}^{-1}$ increase the functional $\mathcal{P}$. In a multidimensional setting, this is clearly not the case.

In Section 4, we prove that the function $\mathcal{P}$ is equivalent to the Besov space $\dot{B}^{0,1,1}$ (17), and we list several equivalent norms. In particular a norm which shows that this space is the dual space of a Banach space of Hölder like functions, and a norm which is related to optimal transport and a degenerate free discontinuity problems.

In Section 5 , we list some properties of the space $B^{0,1,1}$. We prove that $\dot{B}^{0,1,1}$ can be represented as a dual space, and that a coarea formula holds. Finally, using some ideas from image reconstruction, we give another equivalent norm.

2. Quasi incompressible BV maps on $\mathbb{R}$. We consider left continuous BV maps $\mathbf{T}: \mathbb{R} \rightarrow \mathbb{R}$, with the following properties

1. $\mathbf{T}$ is invertible,

2. $\mathbf{T}-\mathbf{I}, \mathbf{T}^{-1}-\mathbf{I}$ are of bounded variation.

For any function $u: \mathbb{R} \rightarrow \mathbb{R}$, we define the advective transport $\mathbf{T} u$ by

$$
\mathbf{T} u(x)=u\left(\mathbf{T}^{-1} x\right) .
$$

Here and in what follows $\mathbf{I}$ is the identity map, $\mathbf{I}(x)=x$.

Similarly, for a given function $\rho: \mathbb{R} \rightarrow \mathbb{R}$, the conservative transport $\mathbf{T} \sharp \rho$ is the push forward of the measure $\rho d x$,

$$
\int_{a}^{b} \mathbf{T} \sharp \rho d x=\int_{\mathbf{T}^{-1}(a, b)} \rho d x .
$$

In the following we also assume that

3. $\mathbf{T} \sharp d x, \mathbf{T}^{-1} \sharp d x$ are absolutely continuous w.r.t. the Lebesgue measure.

This implies that the derivatives of $\mathbf{T}, \mathbf{T}^{-1}$ do not have the Cantorian part and that

$$
\mathbf{T} \sharp \rho(x)=\left|\frac{d \mathbf{T}^{-1}(x)}{d x}\right| \rho\left(\mathbf{T}^{-1}(x)\right)=\left|\frac{d \mathbf{T}\left(\mathbf{T}^{-1}(x)\right)}{d x}\right|^{-1} \rho\left(\mathbf{T}^{-1}(x)\right)
$$


outside the countable jump set of $\mathbf{T}^{-1}$. If

$$
\left|\frac{d \mathbf{T}^{-1}}{d x}\right|=1
$$

then $\mathbf{T}$ is measure preserving. Clearly also $T^{-1}$ is measure preserving.

LEMma 1. If $\mathbf{T}: \mathbb{R} \rightarrow \mathbb{R}$ satisfies conditions 1), 2), 3) above, then by a change of variable we can assume $\mathbf{T}$ to be measure preserving.

Proof. We can perform the following change of variable: if $\mathbf{T}$ is as above, define the variable

$$
z(y)=(\mathbf{T} \sharp d x)(0, y)=\int_{0}^{y}(\mathbf{T} \sharp 1) d s,
$$

and the map $\widetilde{\mathbf{T}}$ by

$$
\widetilde{\mathbf{T}}(x)=z(\mathbf{T}(x)) .
$$

It thus follows that

$$
\begin{aligned}
\widetilde{\mathbf{T}} \sharp \rho(x) & =\left|\frac{d \widetilde{\mathbf{T}}^{-1}(x)}{d x}\right| \rho\left(\widetilde{\mathbf{T}}^{-1}(x)\right) \\
& =\left|z^{\prime}\left(\mathbf{T}\left(\widetilde{\mathbf{T}}^{-1}(x)\right)\right) \frac{d \mathbf{T}\left(\widetilde{\mathbf{T}}^{-1}(x)\right)}{d x}\right|^{-1} \rho\left(\widetilde{\mathbf{T}}^{-1}(x)\right) \\
& =\left|\left(\frac{d \mathbf{T}\left(\widetilde{\mathbf{T}}^{-1}(x)\right)}{d x}\right)^{-1} \frac{d \mathbf{T}\left(\widetilde{\mathbf{T}}^{-1}(x)\right)}{d x}\right|^{-1} \rho\left(\widetilde{\mathbf{T}}^{-1}(x)\right) \\
& =\rho\left(\widetilde{\mathbf{T}}^{-1}(x)\right) .
\end{aligned}
$$

Thus the map $\widetilde{\mathbf{T}}$ is measure preserving.

In the following we will study a sequence of maps $\mathcal{T}=\left\{\mathbf{T}_{i}\right\}_{i=1}^{\infty}$. We say that the sequence of maps $\left\{\mathbf{T}_{i}\right\}_{i=1}^{\infty}$ is quasi incompressible if the functions

$$
\rho_{i+1}=\mathbf{T}_{i} \sharp \rho_{i}, \quad \rho_{0} \equiv 1,
$$

satisfy the uniform bound

$$
\frac{1}{C_{1}} \leq \rho_{i} \leq C_{1}, \quad C_{1} \in[1,+\infty),
$$

with $C_{1}$ independent on $i \in \mathbb{N}$.

We assume that the sequence $\mathcal{T}$ satisfies properties 1), 2), 3) and moreover that

4. the sequence $\mathcal{T}$ is quasi incompressible;

5. the total variation of $\mathbf{T}_{i}-\mathbf{I}$ and $\mathbf{T}_{i}^{-1}-\mathbf{I}$ are summable:

$$
\sum_{i=1}^{\infty}\left(\operatorname{Tot} . \operatorname{Var} .\left(\mathbf{T}_{i}-\mathbf{I}\right)+\operatorname{Tot} . \operatorname{Var} .\left(\mathbf{T}_{i}^{-1}-\mathbf{I}\right)\right)=\mathcal{T} \mathcal{V}(\mathcal{T}) \leq+\infty
$$

As in the proof of Lemma 1 , by defining the sequence of variables $y_{i}$ and maps $\widetilde{\mathbf{T}}_{i}$ by

$$
y_{i}(x)=\int_{0}^{x}\left(\mathbf{T}_{i} \sharp 1\right) d x, \quad \widetilde{\mathbf{T}}_{i}(x)=y_{i}\left(\left(\mathbf{T}_{i}\right)(x)\right),
$$


it follows from (31) that $y=y_{i} \circ y_{i-1} \circ \ldots \circ y_{1}(x)$ is an invertible map for all $i$,

$$
\frac{1}{C_{1}} \leq \frac{d y}{d x} \leq C_{1} .
$$

Moreover the maps $\widetilde{\mathcal{T}}=\left\{\widetilde{\mathbf{T}}_{i}\right\}_{i=1}^{\infty}$ are measure preserving:

$$
\widetilde{\mathbf{T}}_{i} \sharp \rho\left(y_{i}\right)=\rho\left(\widetilde{\mathbf{T}}_{i}^{-1} y_{i}\right) .
$$

In this case, each map $\widetilde{\mathbf{T}}_{i}$ has only jumps and a continuous derivative equal to 0 or -2 .

We consider now the following functional on characteristic functions on $\mathbb{R}$ : if $\mu$ is a measure on $\mathbb{R}$ such that $\mu(a, b) \neq 0$ for all $a<b$, then

$$
\mathcal{P}(A ; \mu)=\int_{0}^{1} \frac{1}{h} \int_{\mathbb{R}}\left|\chi_{A}(x)-\frac{1}{\mu(x-h, x+h)} \int_{x-h}^{x+h} \chi_{A}(y) d \mu(y)\right| d \mu(x) d h .
$$

For the sake of generality, we have defined the functional for a general measure $\mu$, but is the following we will use only $\mathcal{P}(A, d x)$. For brevity, we will write $\mathcal{P}(A, d x)=\mathcal{P}(A)$,

$$
\mathcal{P}(A)=\int_{0}^{1} \frac{1}{h} \int_{\mathbb{R}}\left|\chi_{A}(x)-\frac{1}{2 h} \int_{x-h}^{x+h} \chi_{A}(y) d y\right| d x d h .
$$

The BV perimeter of a set is denoted by $P(A)$.

Consider now a function $\rho \in L^{\infty}$ with values in $\left[1 / C_{1}, C_{1}\right]$, and define

$$
y(x)=\int_{0}^{x} \rho d x .
$$

We want to compare $\mathcal{P}(A)$ with

$$
\mathcal{P}^{\prime}(A)=\int_{0}^{1} \frac{1}{h} \int_{\mathbb{R}}\left|\chi_{A}\left(y^{-1}(x)\right)-\frac{1}{2 h} \int_{x-h}^{x+h} \chi_{A}\left(y^{-1}(z)\right) d z\right| d x d h .
$$

for a measurable set $A$. If we denote by $I(x, h ; A), I^{\prime}(x, h ; A)$ the integrals

$$
\begin{aligned}
I(x, h ; A) & =\frac{1}{2 h} \int_{x-h}^{x+h} \chi_{A}(z) d z, \\
I^{\prime}(x, h ; A) & =\frac{1}{2 h} \int_{x-h}^{x+h} \chi_{A}\left(y^{-1}(z)\right) d z,
\end{aligned}
$$

then it follows that, independently of $A$,

$$
\begin{aligned}
I^{\prime}(x, h ; A) & \leq C_{1} \frac{1}{2 h} \int_{y^{-1}(x-h)}^{y^{-1}(x+h)} \chi_{A}(z) d z \\
& \leq C_{1} \frac{1}{2 h} \int_{y^{-1}(x)-C_{1} h}^{y^{-1}(x)+C_{1} h} \chi_{A}(z) d z \leq C_{1}^{2} I\left(y^{-1}(x), C_{1} h ; A\right) .
\end{aligned}
$$

We thus have the estimate

$$
\begin{aligned}
\mathcal{P}^{\prime}(A) & =\int_{0}^{1} \frac{1}{h} \int_{\mathbb{R}}\left|\chi_{A}\left(y^{-1}(x)\right)-\frac{1}{2 h} \int_{x-h}^{x+h} \chi_{A}\left(y^{-1}(z)\right) d z\right| d x d h \\
& \leq C_{1}\left\{\int_{0}^{1} \frac{1}{h} \int_{\mathbb{R}}\left|\chi_{A}(x)-\frac{1}{2 h} \int_{y(x)-h}^{y(x)+h} \chi_{A}\left(y^{-1}(z)\right) d z\right| d x d h\right.
\end{aligned}
$$




$$
\begin{aligned}
= & C_{1}\left\{\int_{0}^{1} \frac{1}{h}\left(\int_{A} I^{\prime}(y(x), h ; \mathbb{R} \backslash A) d x+\int_{\mathbb{R} \backslash A} I^{\prime}(y(x), h ; A) d x\right) d h\right\} \\
\leq & C_{1}^{3}\left\{\int_{0}^{1} \frac{1}{h}\left(\int_{A} I\left(x, C_{1} h ; \mathbb{R} \backslash A\right) d x+\int_{\mathbb{R} \backslash A} I\left(x, C_{1} h ; A\right) d x\right) d h\right\} \\
= & C_{1}^{3} \int_{0}^{1} \frac{1}{h} \int_{\mathbb{R}}\left|\chi_{A}(x)-I(x, h ; A)\right| d x d h \\
& +C_{1}^{3} \int_{1}^{C_{1}} \frac{1}{h} \int_{\mathbb{R}}\left|\chi_{A}\left(y^{-1}(x)\right)-I\left(y^{-1}(x), h ; A\right)\right| d x d h .
\end{aligned}
$$

We prove the following lemma, which allows one to compare the integral

$$
\int_{\mathbb{R}}\left|\chi_{A}(x)-\frac{1}{2 h} \int_{x-h}^{x+h} \chi_{A}(y) d y\right| d x
$$

for different values of $h$.

LEMMA 2. We have

$$
\begin{aligned}
\int_{\mathbb{R}}\left|\frac{1}{3 h} \int_{x-3 h / 2}^{x+3 h / 2}\left(\chi_{A}(x)-\chi_{A}(z)\right) d z\right| d x \\
\leq \frac{16}{3} \int_{\mathbb{R}}\left|\frac{1}{2 h} \int_{x-h}^{x+h}\left(\chi_{A}(x)-\chi_{A}(z)\right) d z\right| d x
\end{aligned}
$$

Proof. We observe that

$$
\frac{1}{2} \chi_{[-3 / 2,3 / 2]}(x) \leq \phi(x) \doteq 2 \chi_{[-1,1]} * \chi_{[-1,1]}(x)= \begin{cases}x+2, & x \in[-2,0] \\ 2-x, & x \in[0,2] \\ 0, & \text { otherwise }\end{cases}
$$

so that, since $\chi_{A}(x)-\chi_{A}(y)$ has a definite sign,

$$
\begin{aligned}
& \left.\left|\frac{1}{3 h} \int_{x-3 h / 2}^{x+3 h / 2}\left(\chi_{A}(x)-\chi_{A}(z)\right) d z\right| \leq 2 \mid \frac{1}{3 h} \int_{\mathbb{R}} \phi((z-x) / h)\left(\chi_{A}(x)-\chi_{A}(z)\right)\right) d z \mid \\
& \quad=\frac{8}{3}\left|\chi_{A}(x)-\frac{1}{2 h} \int_{x-h}^{x+h} \chi_{A}(z) d z+\frac{1}{2 h} \int_{x-h}^{x+h}\left(\chi_{A}(y)-\frac{1}{2 h} \int_{y-h}^{y+h} \chi_{A}(z) d z\right)\right|,
\end{aligned}
$$

so that the conclusion follows by integrating in $x$.

By Lemma 2, it follows that for some constant $C_{2}$, independent of $A$, we have

$$
\int_{1}^{C_{1}} \frac{1}{h} \int_{\mathbb{R}}\left|\chi_{A}\left(y^{-1}(x)\right)-I\left(y^{-1}(x), h ; A\right)\right| d x d h \leq C_{2} \int_{0}^{1} \frac{1}{h} \int_{\mathbb{R}}\left|\chi_{A}(x)-I(x, h ; A)\right| d x d h .
$$

This clearly implies that the functionals $\mathcal{P}(A), \mathcal{P}^{\prime}(A)$ are equivalent,

$$
\frac{1}{C_{3}} \mathcal{P}(A) \leq \mathcal{P}^{\prime}(A) \leq C_{3} \mathcal{P}(A)
$$

where the constant $C_{3}$ is independent on $A$.

We can use this result to deal with only divergence free maps. In fact, by the assumption of quasi incompressibility, after the change of variable $y_{i}(x)$ the maps $\left\{\widetilde{T}_{i}\right\}_{i=1}^{\infty}$ of (33) are measure preserving, and their corresponding functionals $\mathcal{P}_{i}(A)$ are equivalent: there 
exists a constant $C_{4}$ such that

$$
\begin{aligned}
\frac{1}{C_{4}} \mathcal{P}(A) & \leq \mathcal{P}_{i}(A)=\int_{0}^{1} \frac{1}{h} \int_{\mathbb{R}}\left|\chi_{A}\left(y_{i}^{-1}(x)\right)-\frac{1}{2 h} \int_{x-h}^{x+h} \chi_{A}\left(y_{i}^{-1}(z)\right) d z\right| d x d h \\
& \leq C_{4} \mathcal{P}(A),
\end{aligned}
$$

independently of $A$. Thus we have proved the following proposition:

Proposition 3. Consider the sequence of maps $\mathcal{T}=\left\{\mathbf{T}_{i}\right\}_{i=1}^{\infty}$. Assume that each $\mathbf{T}_{i}$ satisfies assumption 1), 2), 3), and that the sequence $\mathcal{T}$ is quasi incompressible. Let $\widetilde{\mathcal{T}}$ be the sequence of maps defined by (33). Given a set $A$, let $A_{i}, \widetilde{A}_{i}$ be the sets defined by

$$
\chi_{A_{i}}=\mathbf{T}_{i} \chi_{A_{i-1}}, \quad \chi_{\widetilde{A}_{i}}=\widetilde{\mathbf{T}}_{i} \chi_{\widetilde{A}_{i-1}}, \quad A_{0}=A .
$$

Then there is a constant $C_{4}$ independent of $A$ such that for all $i$

$$
\frac{1}{C_{4}} \mathcal{P}\left(A_{i}\right) \leq \mathcal{P}\left(\widetilde{A}_{i}\right) \leq C_{4} \mathcal{P}\left(A_{i}\right)
$$

The constant $C_{4}$ depends only on the quasi incompressibility constant of the sequence $\mathcal{T}$.

3. Divergence free maps. By Proposition 3, to estimate the increase of $\mathcal{P}(A)$ under the action of $\mathcal{T}=\left\{\mathbf{T}_{i}\right\}_{i=1}^{\infty}$, it is sufficient to estimate $\mathcal{P}(A)$ under the action of $\widetilde{\mathcal{T}}=\left\{\widetilde{\mathbf{T}}_{i}\right\}$ given by (33). Moreover, Tot.Var. $\left(\widetilde{\mathbf{T}}_{i}-\mathbf{I}\right)$ is of the same order of Tot.Var. $\left(\mathbf{T}_{i}-\mathbf{I}\right)$ (their ratio is of the order of the constant of quasi incompressibility). It is thus sufficient to work with measure preserving maps, and to avoid cumbersome notation we will neglect the tilde.

We now estimate the difference between $\mathcal{P}(A)$ and $\mathcal{P}(\mathbf{T} A)$, where $A$ is a measurable set. We want to prove that this quantity is of the order of

$$
\text { Tot.Var. }(\mathbf{T}-\mathbf{I})+\text { Tot.Var. }\left(\mathbf{T}^{-1}-\mathbf{I}\right) \text {. }
$$

More precisely, we will show that this difference is controlled by the measure of the jump part of $\mathbf{T}-\mathbf{I}, \mathbf{T}^{-1}-\mathbf{I}$ (which is also the jump part of $\mathbf{T}, \mathbf{T}^{-1}$ ). We remark again that this is a particular feature of the one-dimensional case.

We can write, by the measure preserving property of $\mathbf{T}$,

$$
\begin{aligned}
\mathcal{P}(\mathbf{T} A)-\mathcal{P}(A)= & \int_{0}^{1} \frac{1}{h} \int_{\mathbb{R}}\left|\chi_{\mathbf{T} A}(x)-\frac{1}{2 h} \int_{x-h}^{x+h} \chi_{\mathbf{T} A}(y) d y\right| d x d h \\
& -\int_{0}^{1} \frac{1}{h} \int_{\mathbb{R}}\left|\chi_{A}(x)-\frac{1}{2 h} \int_{x-h}^{x+h} \chi_{A}(y) d y\right| d x d h \\
= & \int_{0}^{1} \frac{1}{h} \int_{\mathbb{R}}\left|\chi_{A}(x)-\frac{1}{2 h} \int_{\mathbf{T}^{-1}(\mathbf{T} x+(-h, h))} \chi_{A}(y) d y\right| d x d h \\
& -\int_{0}^{1} \frac{1}{h} \int_{\mathbb{R}}\left|\chi_{A}(x)-\frac{1}{2 h} \int_{x-h}^{x+h} \chi_{A}(y) d y\right| d x d h \\
\leq & \int_{0}^{1} \frac{1}{h} \int_{\mathbb{R}}\left|\frac{1}{2 h} \int_{\mathbf{T}^{-1}(\mathbf{T} x+(-h, h))} \chi_{A}(y) d y-\frac{1}{2 h} \int_{x-h}^{x+h} \chi_{A}(y) d y\right| d x d h \\
\leq & \int_{0}^{1} \frac{1}{2 h^{2}} \int_{\mathbb{R}}\left(2 h-\left|\mathbf{T}^{-1}(\mathbf{T} x+(-h, h)) \cap(x+(-h+h))\right|\right) d x d h .
\end{aligned}
$$


It is thus sufficient to compute the length of the set $\mathbf{T}(x+(-h, h))$ which is mapped outside the segment $\mathbf{T} x+(-h, h)$.

Let $a$ be the first point in $(-h, h)$ not mapped by $\mathbf{T}$ in $(-h, h)$, and assume for simplicity that $a>0$. By conservation of measure, it follows that $\mathbf{T}^{-1}$ maps a region of length $h-a$ of the segment $\left(0, \mathbf{T}\left(a^{-}\right)\right)$outside $(0, a)$. Then $\mathbf{T}^{-1}$ has at least a jump of size $(h-a) / 2$.

If $2 b$ is the total length of the set mapped outside, i.e.

$$
2 b=2 h-\left|\mathbf{T}^{-1}(\mathbf{T} x+(-h, h)) \cap(x+(-h+h))\right|,
$$

then it follows that either $\mathbf{T}$ or $\mathbf{T}^{-1}$ has at least a jump of size $(h-b / 2) / 2$ : in fact, in the best case the point $h-b / 2$ is mapped outside $(-h, h)$.

We can now estimate the difference for each fixed $x, h$ by

$$
\begin{aligned}
\Delta(x, h) & =2 h-\left|\mathbf{T}^{-1}(\mathbf{T} x+(-h, h)) \cap(x+(-h+h))\right| \\
& \leq 2 \min \left\{h, \text { greater jump of } \mathbf{T}, \mathbf{T}^{-1} \text { in } x+(-h, h)\right\} .
\end{aligned}
$$

This inequality tells us that the worst case is when two jumps of size $h$ are located at $-h, h$.

Consider a jump of size $\ell$ of $\mathbf{T}$, and a segment $x+(-h, h)$. We say that the jump is effective in $x+(-h, h)$ if the size of length mapped outside is less or equal to $2 \ell$. In fact, if $\Delta(x, h)$ is greater than this, then we know from (45) that there is a larger jump which is effective. We thus have the following estimates:

1. if $h \leq 2 \ell$, then it follows that the jump moves a segment of length at most $h$ outside $\mathbf{T} x+(-h, h)$ for all $x$ at a distance $h$ of the location of the jump;

2. if $h>2 \ell$, then the jump is effective only when it is near the boundary of $x+(-h, h)$, at a distance of at most $2 \ell$. In fact in the other cases there is a bigger jump which we can take into account to estimate the length difference. It thus follows that it can influence a region of length $4 \ell$, and the length of the region moved outside is of order $\ell$, because we assume it is effective.

The same estimates hold for the jumps in $\mathbf{T}^{-1}$.

We thus have

$$
\begin{aligned}
\int_{\mathbb{R}}|\Delta(x, h)| d x & \leq h^{2} \sharp\left\{\text { jumps of } T, T^{-1} \text { of size } \geq h / 2\right\}+\sum_{\text {size } \leq h / 2} \mid \text { size of the jump }\left.\right|^{2} \\
& \leq h^{2} \sharp\left\{\text { jumps of } T, T^{-1}\right\}+\sum_{\text {size } \leq h / 2} \mid \text { size of the jump }\left.\right|^{2},
\end{aligned}
$$

and we conclude that

$$
\begin{aligned}
|\mathcal{P}(\mathbf{T} A)-\mathcal{P}(A)| & \leq \int_{0}^{1} \frac{1}{2 h^{2}}\left(h^{2} \sharp\left\{\text { jumps of } T, T^{-1}\right\}\right. \\
& \left.+\sum_{\text {size } \leq h / 2} \mid \text { size of the jump }\left.\right|^{2}\right) d h \\
& \leq C_{4} \sum_{\text {jumps }}\left(\int_{0}^{\text {size }} d h+|\operatorname{size}|^{2} \int_{\text {size }}^{1} \frac{d h}{h^{2}}\right) \leq C_{5} \sum_{\text {jumps }} \mid \text { size } \mid \\
& \leq C_{5}\left(\operatorname{Tot} . \operatorname{Var} .(\mathbf{T}-\mathbf{I})+\operatorname{Tot} . \operatorname{Var} .\left(\mathbf{T}^{-1}-\mathbf{I}\right)\right) .
\end{aligned}
$$




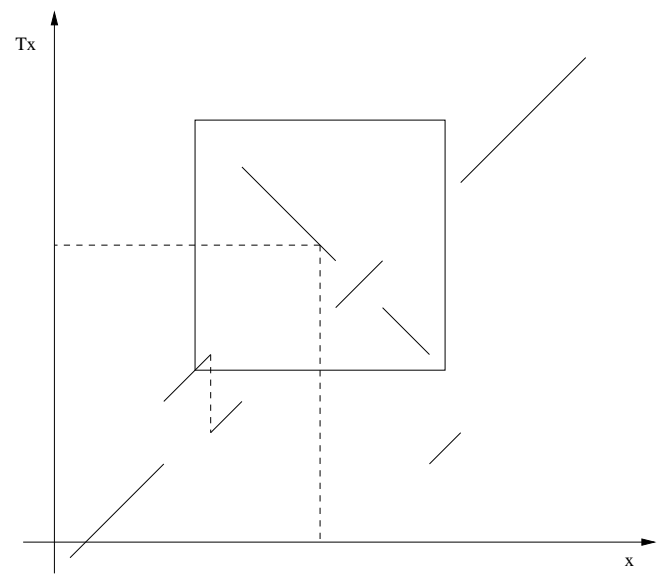

Fig. 1. The influence of a jump affects only a small set when $h$ is large

This result proves the following theorem:

THEOREM 4. Let $\mathcal{T}$ be a sequence of maps satisfying 1), 2), 3), 4), 5). Let $A_{i}$ be the sets defined by

$$
\chi_{A_{i}}=\mathbf{T}_{i} \chi_{A_{i-1}}, \quad A_{0}=A .
$$

Then there exists a positive constant $C_{5}$, independent of $A$ such that

$$
\mathcal{P}\left(A_{i}\right) \leq \mathcal{P}(A)+C_{5} \mathcal{T} \mathcal{V}(\mathcal{T})
$$

3.1. Application to Bressan's conjecture. The result stated in Theorem 4 is a generalization to quasi incompressible BV maps on $\mathbb{R}$ of Bressan's result on mixing properties of the BV maps (8).

To show the logarithmic estimate, it is enough to compute $\mathcal{P}(A)$ for a set $A$ mixed up to scale $\bar{h}$, i.e such that

$$
\frac{1}{2 \bar{h}} \int_{x-\bar{h}}^{x+\bar{h}} \chi_{A}(y) d y \in(c, 1-c) .
$$

With simple estimates, it follows that for all $h \geq \bar{h}$ we have

$$
\frac{1}{2 h} \int_{x-h}^{x+h} \chi_{A}(y) d y \in(c / 2,1-c / 2) .
$$

Then we can estimate

$$
\mathcal{P}(A) \geq|A| \frac{c}{2} \log \frac{1}{h} .
$$

We thus conclude that

$$
\text { Tot.Var. }(\mathbf{T}-\mathbf{I})+\text { Tot.Var. }\left(\mathbf{T}^{-1}-\mathbf{I}\right) \geq \mathcal{O}(1) \log \frac{1}{h},
$$

which is the same estimate given by Bressan [9].

4. Definition of the Besov space $B^{0,1,1}$ in $\mathbb{R}^{n}$. In this section we show that the function $\mathcal{P}$ is equivalent to the homogeneous norm in the Besov space $B^{0,1,1}$ for characteristic 
functions of measurable sets. This space is the space of measurable functions from $\mathbb{R}^{n}$ to $\mathbb{R}$ with the norm

$$
\|u\|_{B^{0,1,1}}=\|u\|_{L^{1}}+\int_{0}^{1} \frac{1}{h} \sup _{|t| \leq h}\left\{\int_{\mathbb{R}^{n}}|u(x+t)-u(x)| d x\right\} d h .
$$

The homogeneous Besov space $B^{0,1,1}$ is the space of measurable functions from $\mathbb{R}^{n}$ to $\mathbb{R}$ with the norm

$$
\|u\|_{\dot{B}^{0,1,1}}=\int_{0}^{1} \frac{1}{h} \sup _{|t| \leq h}\left\{\int_{\mathbb{R}^{n}}|u(x+t)-u(x)| d x\right\} d h .
$$

In the following for simplicity we use the notation

$$
u_{t}(x)=u(x+t) .
$$

REMARK 5. Observe that since this space is based on $L^{1}$, the definition of $B^{0,1,1}$ in terms of Littlewood-Paley decomposition

$$
\|u\|=\sum_{i=1}^{\infty}\left\|\phi_{i} * u\right\|_{L^{1}}, \quad \phi_{i}(x)=\left(2^{i}\right)^{n} \phi\left(2^{i} x\right),
$$

is not equivalent to (51). The function $\phi$ is the function used in the Fourier decomposition, i.e. its Fourier transform $\hat{\phi}$ has the form

$$
\hat{\phi}(\xi)=\psi(\xi / 2)-\psi(\xi),
$$

and $\psi$ is a smooth function such that $\psi=1$ on $|\xi| \leq 1 / 2, \psi=0$ for $|\xi| \geq 1$.

We first enumerate some elementary properties of these spaces.

1. If $\|u\|_{\dot{B}^{0,1,1}}<\infty$ then $u \in L_{\mathrm{loc}}^{1}$.

2. If $u \in \mathrm{BV}$, then $u \in \dot{B}^{0,1,1}$.

3. The space $B^{0,1,1}$ is a Banach space with norm (51), compactly embedded into $L^{1}$.

The first two statements are trivial. To prove the last assertion, we first observe that if $\omega(h ; u)$ is the modulus of continuity of $u$,

$$
\omega(h ; u)=\sup _{|t| \leq h}\left\{\int_{\mathbb{R}^{n}}|u(x+t)-u(x)| d x\right\},
$$

(or the concave modulus of continuity), then we can rewrite (52) as

$$
\|u\|_{\dot{B}^{0,1,1}}=\int_{0}^{1} \omega(h ; u) \frac{d h}{h} .
$$

The compactness of this space follows by standard results on compact sets in $L^{1}$.

In the following proposition we give equivalent norms on the space $\dot{B}^{0,1,1}$, other than (58).

Proposition 6. The norm (52) is equivalent to the following quantities:

$$
\begin{gathered}
\int_{0}^{1} \omega(h, u) \frac{d h}{h} ; \\
\frac{1}{|B(0,1)|} \int_{B(0,1)} \frac{1}{|h|^{n}} \int_{\mathbb{R}^{n}}|u(x+h)-u(x)| d x d h ;
\end{gathered}
$$




$$
\begin{aligned}
& \int_{0}^{1} \sup \left\{\int u(x) \operatorname{div} \psi(x) d x ;|\psi(x)|, h|\operatorname{div} \psi(x)| \leq 1\right\} d h \\
& \|\| u \| \doteq \int_{0}^{1} \min \left\{\operatorname{Tot} . \operatorname{Var} .(v)+\frac{\|u-v\|_{L^{1}}}{h}, v \in \mathrm{BV}\right\} d h .
\end{aligned}
$$

The minimum in the above equation means that there is a $v_{h} \in \mathrm{BV}$ such that the infimum is assumed.

Moreover, if $u$ is the characteristic function of a measurable set $A$, then the homogeneous Besov norm is equivalent to the functional $\mathcal{P}(A)$,

$$
\mathcal{P}(A)=\int_{0}^{1} \frac{1}{h} \int_{\mathbb{R}^{n}}\left|\chi_{A}(x)-\frac{1}{|B(0, h)|} \int_{B(x, h)} \chi_{A}(y) d y\right| d x d h .
$$

Proof. The first is just the definition. For the second one we have

$$
\begin{aligned}
\int_{B(0,1)} \frac{1}{|h|^{n}} \int_{\mathbb{R}^{n}}|u(x+h)-u(x)| d x d h \\
\leq \int_{B(0,1)} \frac{1}{|h|^{n}} \sup _{|t| \leq h}\left\{\int_{\mathbb{R}^{n}}|u(x+t)-u(x)| d x\right\} d h \\
\quad=\int_{0}^{1} \frac{|\partial B(0,1)|}{|h|} \sup _{|t| \leq h}\left\{\int_{\mathbb{R}^{n}}|u(x+t)-u(x)| d x\right\} d|h| .
\end{aligned}
$$

Conversely, one can use the triangular estimate

$$
\left\|u_{t}-u\right\|_{L^{1}} \leq \sum_{i}\left\|u_{t_{i}}-u\right\|_{L^{1}}, \quad \sum_{i} t_{i}=t
$$

For fixed $t \in B(0, h)$, the set such that the above second equation holds has clearly positive measure in $\{|t| \leq h\}$, so that by integrating we have for some constant $C>0$ independent of $h$

$$
\sup _{|t| \leq h}\left\|u_{t}-u\right\|_{L^{1}} \leq \frac{C}{h^{n}} \int_{|t| \leq h}\left\|u_{t}-u\right\|_{L^{1}} d t .
$$

By integration by parts it follows

$$
\begin{aligned}
\int_{0}^{1} \frac{1}{h} \sup _{|t| \leq h}\left\|u_{t}-u\right\|_{L^{1}} d h & \leq \int_{0}^{1} \frac{C}{h^{n+1}} \int_{|t| \leq h}\left\|u_{t}-u\right\|_{L^{1}} d t d h \\
& \leq C_{1} \int_{|t| \leq 1} \frac{1}{|t|^{n}}\left\|u_{t}-u\right\|_{L^{1}} d t .
\end{aligned}
$$

To prove the equivalence of the norm (52) with (59), (60), we prove first that $\|u\|_{\dot{B}^{0,1,1}}$ is bounded by (59) by choosing

$$
\psi(x)=\left(\int_{0}^{x}(\operatorname{sgn}(u(x)-u(x-h))-\operatorname{sgn}(u(x+h)-u(x))) d x, 0, \ldots, 0\right),
$$

and observing that clearly $|\psi| \leq 1$.

Next we prove that (59) is equal to (60). We consider the representation of BV as a dual space: let

$$
X=\left\{\left(\phi_{1}, \ldots, \phi_{n}\right) \in\left(C_{0}\left(\mathbb{R}^{n}, \mathbb{R}\right)\right)^{n}\right\}, \quad Y=\bar{E},
$$


where the norm of $X$ is the usual sup norm, and the set $E$ is defined by

$$
E=\left\{\left(\phi_{1}, \ldots, \phi_{n}\right) \in\left(C_{0} \cap C^{1}\left(\mathbb{R}^{n}, \mathbb{R}\right)\right)^{n}: \sum_{i=1}^{n} \frac{\partial \phi_{i}}{\partial x_{i}}=0\right\}
$$

Then it is known that

$$
\dot{B V}=(X / Y)^{*},
$$

where $\dot{B V}$ is the homogeneous BV space with the total variation as norm [5].

The argument is based on the min max principle

$$
\inf _{\psi \in X / Y}\{\Theta(\psi)+\Xi(\psi)\}=\sup _{v \in B V}\left\{-\Theta^{*}(v)-\Xi^{*}(-v)\right\},
$$

where $\Theta, \Xi$ are convex functions and $\Theta^{*}, \Xi^{*}$ their Legendre transforms, and there exists a $\bar{\psi} \in X / Y$ such that $\Theta(\bar{\psi})+\Xi(\bar{\psi})<\infty$ and $\Theta$ is continuous in $\bar{\psi}$. Moreover the supremum in the right hand side is assumed.

We write the integrand (59) as the infimum of the sum of two convex functionals: for $\psi \in X / Y$, we set

$$
\begin{gathered}
\Theta(\psi)= \begin{cases}0, & |\psi| \leq 1, \\
+\infty, & \text { otherwise, }\end{cases} \\
\Xi(\psi, u)= \begin{cases}\int_{\mathbb{R}^{n}} u \operatorname{div} \psi, & \psi \in C^{1},|\operatorname{div} \psi| \leq 1 / h, \\
+\infty, & \text { otherwise. }\end{cases}
\end{gathered}
$$

It is easy to see that by choosing $\bar{\psi}=0$ the conditions needed by (65) are satisfied.

The Legendre transforms are computed easily: for $v \in B V$,

$$
\begin{gathered}
\Theta^{*}(v)=\operatorname{Tot} . \operatorname{Var} .(v), \\
\Xi^{*}(-v)=\sup \left\{\int_{\mathbb{R}^{n}}(u-v) \operatorname{div} \psi:|\operatorname{div} \psi| \leq 1 / h\right\}=\frac{1}{h}\|u-v\|_{L^{1}} .
\end{gathered}
$$

The last equality follows because we are not requiring any bound on the $L^{\infty}$ norm of $\psi$.

Thus by (65) we conclude that

$$
\begin{aligned}
\|u\| & =\int_{0}^{1} \inf \left\{\operatorname{Tot} . \operatorname{Var} .(v)+\frac{1}{h}\|u-v\|_{L^{1}}, v \in \mathrm{BV}\right\} d h \\
& =\int_{0}^{1} \sup \left\{\int u \operatorname{div} \psi d x ;|\psi|, h|\operatorname{div} \psi| \leq 1\right\} d h .
\end{aligned}
$$

Moreover the infimum is attained, i.e. there is a $v_{h} \in \mathrm{BV}$ depending on $h$ such that

$$
\|\mid u\|=\int_{0}^{1} \operatorname{Tot} . \operatorname{Var} .\left(v_{h}\right)+\frac{1}{h}\left\|u-v_{h}\right\|_{L^{1}} d h .
$$

We finally prove that (59) is bounded by (52). Choosing as a particular $v$ the function $\left(\chi_{B(0, h)} / h^{n}\right) * u$, we have

$$
\begin{aligned}
\text { Tot.Var. }\left(\left(\chi_{B(0, h)} / h^{n}\right) * u\right) & =\frac{1}{h} \int_{\mathbb{R}^{n}} \int_{\partial B(x, h)}|u(x+y)-u(x)| d y d x \\
\left\|\left(\chi_{B(0, h)} / h^{n}\right) * u-u\right\|_{L^{1}} & =\int_{\mathbb{R}^{n}} \int_{B(0, h)}|u(x+y)-u(x)| d y d x
\end{aligned}
$$


so that $\|\mid u\|\|\geq\| u \|_{\dot{B}^{0,1,1}}$. This concludes the proof of the proposition for general functions $u$.

For the case where $u=\chi_{A}$, we need only observe that using (58) or its equivalent formulation

$$
\int_{0}^{1} \frac{d h}{h} \iint_{|x-y| \leq h} \frac{|u(x)-u(y)|}{h^{n}} d x d y
$$

we have

$$
\begin{aligned}
\left\|\chi_{A}\right\|_{\dot{B}^{0,1,1}}= & \int_{0}^{1} \frac{d h}{h} \int_{A}\left(\chi_{A}(x)-\frac{1}{|B(0, h)|} \int_{B(x, h)} \chi_{A}(y) d y\right) d x \\
& +\int_{0}^{1} \frac{d h}{h} \int_{\mathbb{R}^{n} \backslash A}\left(\frac{1}{|B(0, h)|} \int_{B(x, h)} \chi_{A}(y) d y\right) d x=\mathcal{P}(A) .
\end{aligned}
$$

This completes the proof.

In the following corollary, we show that to estimate the norm (60), one can choose the function

$$
\rho_{h} * u=\frac{1}{h^{n}} \int_{\mathbb{R}^{n}} \rho\left(\frac{x-y}{h}\right) u(y) d y,
$$

where $\rho$ is a standard convolution kernel. This gives another equivalent norm.

Corollary 7. We have

$$
\begin{aligned}
& \frac{1}{3} \int_{0}^{1} \text { Tot.Var. }\left(\rho_{h} * u\right)+\frac{\left\|u-\rho_{h} * u\right\|_{L^{1}}}{h} d h \leq\|\| u \| \\
& \leq \int_{0}^{1} \text { Tot.Var. }\left(\rho_{h} * u\right)+\frac{\left\|u-\rho_{h} * u\right\|_{L^{1}}}{h} d h .
\end{aligned}
$$

Proof. The second part of the corollary is a consequence of the definition of \|\|$u\|\|$. For the first part one has

$$
\begin{aligned}
& \operatorname{Tot.Var.~}\left(\rho_{h} * u\right) \leq \operatorname{Tot.Var} .\left(\rho_{h} * v\right)+\operatorname{Tot} . \operatorname{Var} .\left(\rho_{h} *(u-v)\right) \\
& \leq \operatorname{Tot.Var} .(v)+\frac{\|u-v\|_{L^{1}}}{h}, \\
& \begin{aligned}
\left\|u-\rho_{h} * u\right\|_{L^{1}} & \leq\|u-v\|_{L^{1}}+\left\|v-\rho_{h} * v\right\|_{L^{1}}+\left\|\rho_{h} *(u-v)\right\|_{L^{1}} \\
& \leq 2\|u-v\|_{L^{1}}+h \operatorname{Tot} . \operatorname{Var} .(v) .
\end{aligned}
\end{aligned}
$$

As a final remark, we observe that the norm ||$|\cdot|||$ resembles a free discontinuity problem when $u$ is the characteristic function of a measurable set: in fact, as we will see later in Proposition 11, for any fixed scale $h$, we are trying to fit $A$ with a smoother set $B$, in such a way as to minimize the difference of area $A \Delta B$, and the cost is the perimeter of $B$ multiplied by $h$.

5. Some properties of $B^{0,1,1}$. In this section we collect some basic property of the Besov space $B^{0,1,1}$. We show that, similarly to $\mathrm{BV}, B^{0,1,1}$ can be considered as the dual space of a particular space of functions. Next we prove a coarea-type formula, and finally a property for the minimization problem given by the equivalent norm (60). 
5.1. $\dot{B}^{0,1,1}$ as a dual space. The same approach used to see $\mathrm{BV}$ as a dual space can be applied here. We first observe that we can rewrite (61) as

$$
\|u\|_{\dot{B}^{0,1,1}}=\int_{0}^{1} \sup \left\{\int_{\mathbb{R}^{n}} u(x) \operatorname{div}_{x} \psi(x) d x: \psi \in X,\|\psi\|_{X} \leq 1\right\} d h,
$$

where $X$ is the space

$$
X=\left\{\psi(x)=\int_{0}^{1} \psi(h, x) d h:\|\psi\|_{X}=\sup _{h \in(0,1]}\left\{\|\psi(h)\|_{C^{0}}+h\left\|\operatorname{div}_{x} \psi(h)\right\|_{C^{0}}\right\}\right\} .
$$

Let $E$ be defined as in (63), and let $Y$ be the closure of $E$ in $X$ using the norm $\|\cdot\|_{X}$. A similar computation as the one done to show that BV is a dual space gives the following proposition:

Proposition 8. The space $\dot{B}^{0,1,1}$ is the dual space of $X / Y$.

5.2. Coarea formula. Since $B^{0,1,1}$ is based on $L^{1}$, it is not surprising that we have a coarea-type formula.

Proposition 9 (Coarea formula). The homogeneous Besov norm

$$
\|u\|_{B^{0,1,1}}=\int_{B(0,1)}\left\|u_{h}-u\right\|_{L^{1}} \frac{d h}{\mid h^{n}}=\iint_{|x-y| \leq 1} \frac{|u(x)-u(y)|}{|x-y|^{n}} d x d y
$$

satisfies the equality

$$
\begin{aligned}
\|u\|_{\dot{B}^{0,1,1}} & =\int_{-\infty}^{+\infty}\|\chi\{u \geq \omega\}\|_{\dot{B}^{0,1,1}} d \omega \\
& =\int_{\mathbb{R}}\left\{\int_{B(0,1)}\left\|\chi\left\{u_{h} \geq \omega\right\}-\chi\{u \geq \omega\}\right\|_{L^{1}} \frac{d h}{|h|^{n}}\right\} d \omega .
\end{aligned}
$$

The proof follows elementarily from the following lemma:

LEMMA 10. We have for $u \in L^{1}$

$$
\begin{aligned}
& \int_{\mathbb{R}^{n}}|u(x+t)-u(x)| d x \\
& \quad=\int_{\mathbb{R}^{n}} \int_{-\infty}^{+\infty}|\chi\{(\omega, x-t): u(x) \geq \omega\}-\chi\{(\omega, x): u(x) \geq \omega\}| d \omega d x .
\end{aligned}
$$

Proof. One can compute directly

$$
\begin{aligned}
u(x+t)-u(x) & =\int_{u(x)}^{u(x+t)} d \omega=\int_{\mathbb{R}}(\chi\{\omega \leq u(x+t)\}-\chi\{\omega \leq u(x)\}) d \omega \\
& =\int_{\mathbb{R}} \chi\{(\omega, x-t): u(x) \geq \omega\}-\chi\{(\omega, x): u(x) \geq \omega\} d \omega,
\end{aligned}
$$

so that the lemma follows.

At this point it is easy to prove the proposition. In fact, the homogeneous Besov norm can be written as

$$
\int_{B(0,1)}\left\|u_{h}-u\right\|_{L^{1}} \frac{d h}{|h|^{n}}=\int_{B(0,1)} \int_{\mathbb{R}}\left\|\chi\left\{u_{h} \geq \omega\right\}-\chi\{u \geq \omega\}\right\|_{L^{1}} d \omega \frac{d h}{|h|^{n}}
$$




$$
\begin{aligned}
& =\int_{\mathbb{R}}\left\{\int_{B(0,1)}\left\|\chi\left\{u_{h} \geq \omega\right\}-\chi\{u \geq \omega\}\right\|_{L^{1}} \frac{d h}{|h|^{n}}\right\} d \omega \\
& =\int_{\mathbb{R}}\|\chi\{u \geq \omega\}\|_{\dot{B}^{0,1,1}} d \omega .
\end{aligned}
$$

Note that by the equivalence of all the norms of Proposition 6 , it follows that the integral of the norm of the level sets of $u$ are equivalent norms. However, also other equivalent norms satisfy the coarea formula.

Proposition 11. The following equality holds:

$$
\begin{aligned}
\int_{0}^{1} \inf \left\{\text { Tot.Var. }(v)+\frac{\|u-v\|_{L^{1}}}{h}\right. & , v \in \mathrm{BV}\} d h \\
& =\int_{\mathbb{R}} \int_{0}^{1} \inf \left\{P(B)+\frac{|\{u \geq \omega\} \Delta B|}{h}\right\} d h d \omega,
\end{aligned}
$$

where $P(B)$ is the $B V$ perimeter of the set $B$ and $A \Delta B=(A \cup B) \backslash(A \cap B)$.

As a corollary, remembering that in the proof of Proposition 6 we actually prove that (59) is equal to (60), we get

Corollary 12. We have

$$
\begin{aligned}
\int_{0}^{1} & \sup \left\{\int u(x) \operatorname{div} \psi(x) d x ;|\psi(x)|, h|\operatorname{div} \psi(x)| \leq 1\right\} d h \\
= & \int_{\mathbb{R}}\left\{\int_{0}^{1} \sup \left\{\int \chi_{u \geq \omega}(x) \operatorname{div} \psi(x) d x ;|\psi(x)|, h|\operatorname{div} \psi(x)| \leq 1\right\} d h\right\} d \omega .
\end{aligned}
$$

The proof of Proposition 11 follows easily from the following lemma:

Lemma 13. For measurable sets $A$,

$$
\int_{0}^{1} \inf \left\{\operatorname{Tot.Var.}(v)+\frac{\left\|\chi_{A}-v\right\|_{L^{1}}}{h}, v \in \mathrm{BV}\right\} d h=\int_{0}^{1} \inf \left\{P(B)+\frac{|A \Delta B|}{h}\right\} d h,
$$

with $P(B)$ the perimeter of $B$.

Proof. Clearly it is enough to prove that

$$
\begin{aligned}
\int_{0}^{1} \inf \left\{P(B)+\frac{|A \Delta B|}{h}\right\} d h \\
\leq \int_{0}^{1} \inf \left\{\operatorname{Tot} . \operatorname{Var} .(v)+\frac{\left\|\chi_{A}-v\right\|_{L^{1}}}{h}, v \in \mathrm{BV}\right\} d h+\epsilon
\end{aligned}
$$

for all $\epsilon>0$. By coarea formula for BV functions, we have

$$
\int_{0}^{1} \operatorname{Tot} . \operatorname{Var} .(v)+\frac{\left\|\chi_{A}-v\right\|_{L^{1}}}{h} d h=\int_{0}^{1}\left\{\int_{0}^{1} P(\{v \geq \omega\})+\frac{|A \Delta\{v \geq \omega\}|}{h} d \omega\right\} d h .
$$

Let $\bar{v}$ be such that

$$
\operatorname{Tot.Var.}(\bar{v})+\frac{\left\|\chi_{A}-\bar{v}\right\|_{L^{1}}}{h} \leq \inf \left\{\operatorname{Tot} . \operatorname{Var} .(v)+\frac{\left\|\chi_{A}-v\right\|_{L^{1}}}{h}, v \in \mathrm{BV}\right\}+\frac{\epsilon}{2},
$$

and $\bar{\omega}$ be such that 


$$
P(\{v \geq \bar{\omega}\})+\frac{|A \Delta\{v \geq \bar{\omega}\}|}{h} \leq \inf _{\omega \in[0,1]}\left\{P(\{v \geq \bar{\omega}\})+\frac{|A \Delta\{v \geq \bar{\omega}\}|}{h}\right\}+\frac{\epsilon}{2}
$$

Then the set $\{v \geq \bar{\omega}\}$ satisfies (83).

5.3. A property of (60). In the norm $(60)$, let $v(h)$ be the minimum of the problem

$$
\inf \left\{\operatorname{Tot.Var} .(v)+\frac{\|u-v\|_{L^{1}}}{h}, v \in \mathrm{BV}\right\} \text {, }
$$

considered for $h>0$. The existence of this minimum follows from the compactness of BV in $L_{\text {loc }}^{1}$.

For $u$ fixed, we define the two functions $a(h ; u), b(h ; u):(0, \infty) \rightarrow \mathbb{R}^{+}$by

$$
a(h ; u)=\operatorname{Tot} . \operatorname{Var} .(v(h)), \quad b(h ; u)=\|u-v(h)\|_{L^{1}} .
$$

We have the following proposition:

Proposition 14. For all bounded sets $A, a\left(h ; \chi_{A}\right),-b\left(h ; \chi_{A}\right)$ are decreasing functions of $h$ and moreover we have

$$
\int_{0}^{+\infty} a\left(h ; \chi_{A}\right) d h=|A| .
$$

Proof. For simplicity, we will write $a(h), b(h)$ instead of $a\left(h ; \chi_{A}\right), b\left(h ; \chi_{A}\right)$. Since

$$
\begin{aligned}
a(h)+\frac{b(h)}{h} & \leq a(h+\epsilon)+\frac{b(h+\epsilon)}{h}, \\
a(h)+\frac{b(h)}{h+\epsilon} & \geq a(h+\epsilon)+\frac{b(h+\epsilon)}{h+\epsilon},
\end{aligned}
$$

we have that

$$
\frac{1}{h}(b(h)-b(h+\epsilon)) \leq a(h+\epsilon)-a(h) \leq \frac{1}{h+\epsilon}(b(h)-b(h+\epsilon)) .
$$

It follows that $b(h)$ is an increasing function of $h$, and that $a(h)$ is decreasing. In particular they are BV functions and by taking their weak derivatives we have

$$
\frac{d a(h)}{d h}+\frac{1}{h} \frac{d b(h)}{d h}=0 .
$$

Noting that $b(0)=0$, and $a(+\infty)=0, b(+\infty)=|A|$, we obtain the formula which gives $a$ as a function of $b$ :

$$
a(h)=\int_{h}^{+\infty} \frac{1}{s} \frac{d b}{d s} d s=-\frac{b(h)}{h}+\int_{h}^{+\infty} \frac{b(s)}{s^{2}} d s .
$$

The result follows by integration in $h$, noting that as $h \rightarrow \infty$ we have $v(h) \rightarrow 0$.

Using the coarea formula, we then deduce

Corollary 15. We have

$$
\int_{0}^{+\infty} a(h ; u) d h=\|u\|_{L^{1}}
$$

Finally, by means of (87), we obtain that the norm (60) becomes

$$
\left\|\chi_{A}\right\| \|=\int_{0}^{1} \frac{b\left(h ; \chi_{A}\right)}{h} d h+\int_{1}^{+\infty} \frac{b\left(h ; \chi_{A}\right)}{h^{2}} d h .
$$

We thus have 
COROllary 16. For sets contained in the ball $B(0,1)$, an equivalent norm of $\dot{B}^{0,1,1}$ is given by

$$
\|u\|_{\dot{B}^{0,1,1}}=\int_{0}^{1} \frac{b\left(h ; \chi_{A}\right)}{h} d h
$$

where $b\left(h ; \chi_{A}\right)$ is defined in (84).

5.4. Application to Bressan's mixing problem. To end this section, we state the following theorem:

TheOREm 17. Let $\mathcal{T}$ be a sequence of invertible maps $\mathbf{T}_{i}$, and assume $\mathcal{T}$ be quasi incompressible. Then for all $n \in \mathbb{N}$ the composed map

$$
\mathbf{S}_{n} \doteq \mathbf{T}_{n} \circ \ldots \circ \mathbf{T}_{1}
$$

is in $\dot{B}^{0,1,1}$ and its norm is bounded by

$$
\left\|\mathbf{S}_{n}\right\|_{\dot{B}^{0,1,1}} \leq C_{6} \sum_{i=1}^{n}\left(\operatorname{Tot} . \operatorname{Var} .\left(\mathbf{T}_{i}-\mathbf{I}\right)+\operatorname{Tot} . \operatorname{Var} .\left(\mathbf{T}_{i}^{-1}-\mathbf{I}\right)\right),
$$

with $C$ a constant depending only on the coefficient of quasi incompressibility.

The proof follows from Theorem 4, Proposition 6 and coarea formula (77).

\section{References}

[1] L. Ambrosio, Transport equation and Cauchy problem for BV vector fields, Invent. Math., 2005, to appear.

[2] L. Ambrosio, F. Bouchut, and C. DeLellis, Well-posedness for a class of hyperbolic systems of conservation laws in several space dimensions, Comm. Partial Differential Equations 29 (2004), 1635-1651.

[3] L. Ambrosio and C. DeLellis, Existence of solutions for a class of hyperbolic systems of conservation laws in several space dimensions, Int. Math. Res. Not., 41 (2003), 2205-2220.

[4] L. Ambrosio and C. DeLellis, On the chain rule for the divergence of BV like vector fields: applications, partial results, open problems, preprint, 2005.

[5] L. Ambrosio, N. Fusco, and D. Pallara, Functions of Bounded Variation and Free Discontinuity Problems, Oxford Mathematical Monographs, 2000.

[6] L. Ambrosio, M. Lecumberry, and S. Maniglia, Lipschitz regularity and approximate differentiability of the DiPerna-Lions flow, preprint, 2005.

[7] F. Bouchut, Renormalized solution to the Vlasov equation with coefficients of bounded variation, Arch. Rational Mech. Anal. 157 (2001), 75-90.

[8] A. Bressan, An ill posed Cauchy problem for a hyperbolic system in two space dimensions, Rend. Sem. Mat. Univ. Padova 110 (2003), 103-117.

[9] A. Bressan, A lemma and a conjecture on the cost of rearrangements, Rend. Sem. Mat. Univ. Padova 110 (2003), 97-102.

[10] G. Crippa and C. DeLellis, Oscillatory solutions to transport equations, to appear, 2005.

[11] C. DeLellis, Blowup of the BV norm in the multidimensional Keyfitz and Kranzer system, Duke Math. J. 127 (2005), 313-339.

[12] N. Depauw, Non-unicité du transport par un champ de vecteurs presque BV, Sémin. Éq. Dériv. Partielles, 2003. 
[13] R. J. DiPerna and P.-L. Lions, Ordinary differential equations, transport theory and Sobolev spaces, Invent. Math. 98 (1989), 511-547.

[14] S. Kruzhkov, First-order quasilinear equations with several space variables, Mat. Sb. 123 (1970), 228-255 (in Russian); English transl.: Math. USSR Sb. 10 (1970), 217-273.

[15] C. LeBris and P.-L. Lions, Renormalized solutions of some transport equations with partially $W^{1,1}$ velocities and applications, Ann. Mat. Pura Appl. 183 (2004), 97-130.

[16] H. Triebel, Theory of Function Spaces, Birkhäuser, 1983. 
